\title{
Audiometria de tronco encefálico (ABR): o uso do mascaramento na avaliação de indivíduos portadores de perda auditiva unilateral
}

Auditory brainstem response (ABR): use of masking in unilateral hearing loss patients

\author{
Melissa M. T. Toma ${ }^{1}$, Carla G. Matas ${ }^{2}$
}

\section{Resumo / Summary}

A meio da $A B R$ ainda é um assunto consideravelmente debatido (Durrant \& Ferraro, 2001). Objetivo: 0 presente estudo propôs investigar a necessidade do mascaramento contralateral, empregado na orelha normal, ao realizar a ABR em indivíduos portadores de perda auditiva neurossensorial unilateral. Forma de estudo: Clínico prospectivo. Material e Método: A amostra constituiu-se de 22 indivíduos portadores de perda auditiva neurossensorial unilateral de grau profundo, sendo 10 do sexo feminino e 12 do sexo masculino, com idades variando entre 9 e 44 anos. Todos os indivíduos foram submetidos a: audiometria tonal liminar, logoaudiometria (SRT, IPRF e SDT), medidas de imitância acústica (incluindo a pesquisa dos reflexos acústicos - modo ipsilateral e contralateral) e audiometria de tronco encefálico na ausência e na presença do mascaramento. Resultados: Todos os indivíduos apresentaram perda auditiva neurossensorial unilateral de grau profundo e curvas timpanométricas do tipo A bilateralmente. Na avaliação da $A B R, 100 \%$ da amostra apresentou presença da Onda $V$ na orelha comprometida, sendo que ao introduzir o mascaramento contralateral tais respostas não foram observadas. Conclusões: 0 mascaramento é um procedimento necessário para a avaliação da audição por meio da ABR em indivíduos portadores de perdas auditivas unilaterais, visando a obtenção de resultados fidedignos. $\mathrm{Na} A B R$, a atenuação interaural para clicks foi maior $(65 \mathrm{~dB})$ do que a observada na audiometria tonal liminar, sendo necessário, portanto, uma menor intensidade de mascaramento para eliminar a resposta da via auditiva contralateral.
Palavras-chave: audição, audiometria de tronco encefálico, mascaramento, perda auditiva unilateral.

Key words: hearing, auditory brainstem response, masking, unilateral hearing loss.
T

he need of masking in auditory brainstem response (ABR) evaluation is still considerably debated issue (Durrant and Ferraro, 2001). Aim: In addition, the present study was to investigate the need of masking in ABR with unilateral hearing loss. Study design: Clinical prospective. Material and Method: The sample was constituded of 22 persons with unilateral hearing loss, being 10 female and 12 male, ranging from 9 to 44 years old. All persons were submited to the following audiological exams: pure - tone and speech audiometry, accoustic impendance tests and audiometry brainstem response in absence and presence of masking. Results: In general, all persons had profound sensorineural unilateral hearing loss and bilateral type - A tympanometric curves. In $A B R$ evaluation, it was possible to observe in $100 \%$ of the sample the presence of wave $V$ in poor ear. As soon as the masking was introduced, these waves has not been viewed. Conclusion: As might be expected that masking must be used with $A B R$ evaluation on unilateral hearing loss to obtain authentical results. In ABR, interaural attenuation to clicks (65 $\mathrm{dB}$ ) was greater the examined in pure-tone audiometry. Thence it follows that less intensity of masking was needed to eliminate the contralateral response.

\footnotetext{
${ }^{1}$ Fonoaudióloga. Especializanda do Curso de Fonoaudiologia da Faculdade de Medicina da Universidade de São Paulo. ${ }^{2}$ Fonoaudióloga. Professora Doutora do Curso de Fonoaudiologia da Faculdade de Medicina da Universidade de São Paulo. Monografia realizada para a conclusão do Curso de Especialização de Fonoaudiologia Clínica do Departamento de Fisioterapia Fonoaudiologia e Terapia O cupacional da Faculdade de Medicina da Universidade de São Paulo.

Endereço para Correspondência: Melissa Mayumi Takahashi Toma - Rua Álvaro Luis Roberto de Assumpção, 79 apto. 61 Campo Belo 04618-020 São Paulo SP. Tel (0xx11) 5531-5084/ 9788-4528 - E-mail: melissa toma@yahoo.com.br

Artigo recebido em 01 de março de 2003. Artigo aceito em 15 de maio de 2003.
} 


\section{INTRODUÇÃO}

A audiometria de tronco encefálico ( $A B R$ ) veio para colaborar em muito na audiologia clínica. É um exame objetivo, de grande sensibilidade, não invasivo e de fácil execução ${ }^{1}$. Sua aplicação possibilita: a avaliação da sensibilidade auditiva de recém-nascidos, crianças ou mesmo adultos nos quais não tenha sido possível realizar um teste confiável, ou mesmo nos casos em que é impossível utilizar outro método; 0 diagnóstico de lesões do nervo auditivo ou tronco encefálico; a monitorização de cirurgias da fossa posterior; a monitorização de pacientes em Centro de Terapia Intensiva, em coma ou sedados, como também avaliação do prognóstico do paciente e auxiliar no diagnóstico da morte encefálica ${ }^{2,3}$.

A ABR refere-se aos potenciais evocados auditivos de curta latência ou potenciais evocados auditivos precoces, cujas respostas ocorrem no intervalo de 0 a 10 milissegundos (ms) iniciais após a apresentação do estímulo acústico.

Este exame consiste em uma série de sete ondas positivas, geradas através de sinais acústicos, que são captadas por meio de eletrodos de superfície conectados à pele. Estas ondas representam a atividade bioelétrica de uma ou mais estruturas ao longo da via auditiva.

De modo geral, as ondas I, III e V são as mais utilizadas, sendo que as outras nem sempre estão presentes na avaliação de indivíduos normais. Estudos normativos realizad os com as latências absolutas e interpicos destas três ondas concluíram que estes valores trazem diversas informações não apenas do limiar de audibilidade, como também do neurodiagnóstico de processos lesivos na via auditiva1,2,3.

$\mathrm{Na}$ avaliação de pacientes que apresentam perdas auditivas unilateral ou bilateral assimétrica, certas precauções são inerentes. $\mathrm{Na}$ audiometria comportamental, quando há uma diferença liminar substancial entre uma orelha e outra, devemos nos atentar à intensidade do estímulo acústico utilizado na testagem da orelha comprometida. Um som intenso, quando apresentado à orelha pior, pode estimular a orelha oposta antes mesmo de atingir o limiar auditivo da orelha testada $a^{4,5}$. Nestas situações, o som pode estimular a orelha contralateral, porém com perda de uma certa quantidade de energia, o que é denominado atenuação interaural ${ }^{5,6}$. No audiograma, um traçado semelhante à curva audiométrica da orelha melhor, porém em um nível mais elevado, será obtido (curva sombra)4.

$\mathrm{Na}$ ocorrência desta situação, é utilizado o mascaramento clínico. Este é um procedimento corriqueiramente empregado na prática da audiologia clínica para obtenção de limiares tonais reais. 0 mascaramento nada mais é que um ruído introduzido na orelha não testada de forma a elevar artificialmente o limiar auditivo desta orelha sem que haja interferências ou influências nos resultados audiométricos da orelha avaliada $4,5,6$.

Ao contrário da audiometria comportamental, a necessidade de mascaramento na avaliação da ABR ainda é um assunto consideravelmente debatido ${ }^{7}$.

Em estudo realizado por Finitzo-Hieber, Hecox \& Cone (1979) não foi encontrada resposta a 110dB NPS (Nível de Pressão Sonora) para clicks avaliando-se a orelha comprometida de indivíduos portadores de perda auditiva unilateral através da ABR. Com esses achados, concluíram que o mascaramento contralateral aplicado na orelha normal não seria necessário na avaliação da audição por meio da $\mathrm{ABR}$, e ainda acrescentaram que o discernimento para não utilizar o mascaramento durante o teste iria torná-lo muito mais curto e rápido. Em outros trabalhos realizados por Galambos \& Hecox (1978) e Owen \& Burkard (1991) observaram que o mascaramento contralateral não influenciava de maneira substancial na avaliação da ABR.

Por outro lado, Chiappa, Gladstone \& Y oung (1979), Ozdamar \& Stein (1981), Humes \& Ochs (1982), Smyth (1985) concluíram em seus estudos que o uso do mascaramento aplicado à orelha não testada seria prudente para a obtenção de respostas eletrofisiológicas confiáveis.

Frente ao exposto, o objetivo do presente estudo é verificar a necessidade do uso do mascaramento contralateral, empregado na orelha normal, ao realizar a ABR em indivíduos portadores de perda auditiva neurossensorial unilateral de grau profundo.

\section{MATERIAL E MÉTODO}

Este estudo foi desenvolvido no Laboratório de Investigação Fonoaudiológica em Potenciais Auditivos Evocados do Centro de Docência e Pesquisa do Departamento de Fisioterapia, Fonoaudiologia e Terapia O cupacional da Faculdade de Medicina da Universidade de São Paulo, durante o período de Outubro a Dezembro de 2002, e aprovado pelo Comitê de Ética do mesmo departamento - 239/02.

Para este estudo foram avaliados 22 indivíduos portadores de perda auditiva neurossensorial unilateral de grau profundo, sendo 10 do sexo feminino, com idade variando de 9 a 44 anos (média $=23$ anos) e 12 do sexo masculino, com idade variando entre 15 e 39 anos (média = 24 anos).

Todos os indivíduos residiam no estado de São Paulo e periodicamente realizavam ou começaram a realizar acompanhamento audiológico no Laboratório de Investigação Fonoaudiológica em Potenciais Auditivos Evocados do Centro de Docência e Pesquisa do Departamento de Fisioterapia, Fonoaudiologia e Terapia Ocupacional da Faculdade de Medicina da Universidade de São Paulo.

Após informações obtidas através da anamnese foi dado início à avaliação audiológica, sendo que os seguintes procedimentos foram empregados:

1- Inspeção do meato acústico externo.

Este procedimento foi realizado com o intuito de verificar 
se havia excesso de cerúmen ou presença de algum corpo estranho que pudessem impedir a passagem do som e conseqüentemente alterar os exames audiológicos subseqüentes.

2- Audiometria tonal liminar e logoaudiometria (SRT - limiar de recepção de fala, IPRF - índice percentual de reconhecimento de fala e SDT - limiar de detecção de fala). Este exame foi realizado com os Audiômetros GSI 10 e GSI 16 - Marca Grason Stadler segundo os critérios adotados por Mangabeira Albernaz et al. (1981);

3- Medidas de imitância acústica compreendidas pela timpanometria, medidas da compliância estática e pesquisa do limiar do reflexo acústico modo contralateral e ipsilateral. As medidas de imitância acústica foram obtidas com 0 Imitanciômetro GSI 33 - Marca Grason Stadler. Para a interpretação dos resultados referentes à curva audiométrica e ao limiar dos reflexos acústicos foram utilizadas, respectivamente, a classificação proposta por Jerger (1970) e Lopes F.․ (1972).

4- Audiometria de tronco encefálico ( $A B R$ ) na ausência e, posteriormente, na presença do mascaramento contralateral.

A ABR foi realizada com o equipamento Modelo Traveler Express da marca Biologic (versão 5.70 modelo 317). Os acessórios ligados ao equipamento foram: fones supra-aurais TDH-39 e eletrodos de platina. 0 estímulo apresentado foi o "click" com polaridade rarefeita, duração de 0,1 milissegundo, velocidade de apresentação de 19,0 clicks/s. Utilizamos a janela de 0 a 10,24 milissegundos e filtros passa baixo e passa alto, respectivamente de $100 \mathrm{~Hz}$ e $3000 \mathrm{~Hz}$. Um total de 2000 estímulos foram testados por duas vezes para analisar a reprodutibilidade do traçado em cada intensidade avaliada. 0 tipo de mascaramento empregado na orelha normal foi o White Noise, sendo 0 mesmo efetivo. A intensidade do mascaramento foi estabelecida de acordo com o limiar eletrofisiológico de cada indivíduo avaliado.

O indivíduo foi acomodado confortavelmente em uma poltrona reclinável. Limpamos sua pele com pasta abrasiva, aplicamos pasta eletrolítica para melhor contato dos eletrodos e estes foram fixos por meio de fita aderente. Três eletrodos foram dispostos de forma a permitirem derivações ipsilaterais: eletrodo ativo (vértex), eletrodo referência (mastóide testada), e eletrodo terra (mastóide não testada). A impedância dos eletrodos foi medida de maneira que se situasse abaixo de $5 \mathrm{k} \Omega$ e o valor da diferença entre um eletrodo e outro de no máximo $2 \mathrm{k} \Omega$.

0 indivíduo foi orientado a manter-se o mais relaxado possível, evitando movimentos bruscos, principalmente da musculatura da região da cabeça e pescoço, que poderiam interferir significantemente no traçado das respostas captadas.

Findados estes procedimentos, iniciamos o exame testando-se a orelha normal a $80 \mathrm{~dB}$ NA para avaliar a integridade da via auditiva e, em seguida, a pesquisa do limiar eletrofisiológico da mesma orelha era realizada. Na avaliação da orelha comprometida foi feita a pesquisa do limiar eletrofisiológico iniciando a ABR na intensidade máxima do estímulo acústico do aparelho ( $90 \mathrm{~dB} N \mathrm{NA})$, pois em alguns casos a $80 \mathrm{~dB}$ NA nenhuma resposta eletrofisiológica podia ser evidenciada. Depois de achados os limiares eletrofisiológicos de ambas as orelhas na ausência do mascaramento, 0 valor da atenuação interaural para clicks era obtido para cada indivíduo e a ABR era novamente testada na presença do mascaramento contralateral.

Para calcularmos o valor inicial do mascaramento aplicado à orelha normal, de cada indivíduo, os seguintes cálculos foram realizados: 1 . 0 valor da atenuação interaural obtido, para clicks, foi subtraído do limiar eletrofisiológico da orelha comprometida; 2 . A este resultado foi somado 10 $\mathrm{dB}$ de intensidade; 3 . 0 resultado deste último cálculo foi a intensidade mínima inicial do mascaramento empregado. Por exemplo, se o limiar eletrofisiológico da orelha normal fosse de $15 \mathrm{~dB}$ NA e da orelha comprometida de $80 \mathrm{~dB}$ NA (na ausência do mascaramento contralateral), a atenuação interaural seria de $65 \mathrm{~dB}$ e o valor inicial do mascaramento aplicado seria de $25 \mathrm{~dB} W N(80 \mathrm{~dB}-65 \mathrm{~dB}+10 \mathrm{~dB})$.

O limiar eletrofisiológico da orelha comprometida foi novamente testado simultaneamente à aplicação do mascaramento contralateral na intensidade anteriormente explicada. Se a resposta permanecesse, a intensidade do mascaramento era aumentada para mais $10 \mathrm{~dB}$ e 0 exame era novamente realizado. Porém, se houvesse ausência de respostas, a intensidade do estímulo acústico (click) era aumentada em mais $10 \mathrm{~dB}$ e os cálculos antes realizados para a aplicação do mascaramento eram novamente computados. Assim, o exame foi realizado até a intensidade máxima do estímulo acústico (clicks) na orelha comprometida ( $90 \mathrm{~dB} N \mathrm{NA}$ ).

Com o intuito de se verificar a necessidade de aplicação do mascaramento, durante a avaliação da audição por meio da ABR, foram realizadas a análise dos resultados obtidos através das seguintes comparações e análises estatísticas: 1. Comparação das respostas eletrofisiológicas das orelhas comprometidas obtidas na ausência do mascaramento contralateral e na presença do mascaramento contralateral 2. Análise da atenuação interaural para clicks 3. Análise da quantidade de mascaramento necessário para que a resposta eletrofisiológica contralateral (da orelha normal) não interferisse nas respostas da orelha comprometida 4. Análise da latência absoluta e amplitude da onda $V$ a 90 dB NA para clicks da orelha comprometida na ausência do mascaramento.

\section{RESULTADOS E DISCUSSÃO}

$\mathrm{Na}$ audiometria tonal liminar, pudemos confirmar a perda auditiva neurossensorial unilateral de grau profundo em $100 \%$ dos indivíduos, sendo $68 \%$ à direita e $32 \%$ à esquerda (Tabela 1). 
Tabela 1. Limiares de audibilidade ( $\mathrm{dB} N \mathrm{NA}$ ) de via aérea obtidos na audiometria tonal liminar dos 22 casos estudados nas frequiências de $250 \mathrm{~Hz}$ a $8000 \mathrm{~Hz}$.

\begin{tabular}{|c|c|c|c|c|c|c|c|c|c|}
\hline Casos & Orelhas & 250 & 500 & 1000 & 2000 & 3000 & 4000 & 6000 & 8000 \\
\hline \multirow[t]{2}{*}{1} & OD & $A R^{*}$ & 115 & 115 & $\mathrm{AR}$ & $\mathrm{AR}$ & $\mathrm{AR}$ & $A R$ & $A R$ \\
\hline & $O E$ & 0 & 0 & 5 & 0 & 0 & 5 & 15 & 15 \\
\hline \multirow[t]{2}{*}{2} & OD & 95 & 95 & 110 & $A R$ & $A R$ & $A R$ & $A R$ & $A R$ \\
\hline & $O E$ & 15 & 10 & 10 & 10 & 0 & 10 & 10 & 5 \\
\hline \multirow[t]{2}{*}{3} & O D & 105 & 105 & 115 & 120 & $A R$ & $A R$ & $A R$ & $A R$ \\
\hline & OE & 15 & 15 & 10 & 5 & 0 & 0 & 15 & 20 \\
\hline \multirow[t]{2}{*}{4} & OD & $A R$ & $A R$ & $A R$ & $A R$ & $A R$ & $A R$ & $A R$ & $A R$ \\
\hline & $O E$ & 10 & 5 & 5 & 5 & 0 & 0 & 5 & 10 \\
\hline \multirow[t]{2}{*}{5} & O D & 5 & 10 & 5 & 5 & 5 & 0 & 10 & 5 \\
\hline & $O E$ & 90 & 95 & $A R$ & $A R$ & $A R$ & $A R$ & $A R$ & $A R$ \\
\hline \multirow[t]{2}{*}{6} & OD & $A R$ & $A R$ & $A R$ & $A R$ & $A R$ & $A R$ & $A R$ & $A R$ \\
\hline & $O E$ & 20 & 10 & 5 & 5 & 0 & 5 & 5 & 0 \\
\hline \multirow[t]{2}{*}{7} & OD & 10 & 5 & 0 & 0 & 5 & 10 & 20 & 25 \\
\hline & $O E$ & $A R$ & AR & $A R$ & $A R$ & $A R$ & $A R$ & $A R$ & $A R$ \\
\hline \multirow[t]{2}{*}{8} & OD & 100 & 105 & 100 & 90 & 95 & 100 & $A R$ & $A R$ \\
\hline & $O E$ & 5 & 10 & 10 & 5 & 5 & 0 & 5 & 5 \\
\hline \multirow[t]{2}{*}{9} & OD & 95 & 100 & 110 & 110 & 110 & 110 & $A R$ & $A R$ \\
\hline & OE & 5 & 5 & 5 & 0 & 0 & 0 & 10 & 0 \\
\hline \multirow[t]{2}{*}{10} & OD & $A R$ & $A R$ & $A R$ & $A R$ & $A R$ & $A R$ & $A R$ & $A R$ \\
\hline & $O E$ & 15 & 0 & 0 & 5 & 15 & 15 & 20 & 10 \\
\hline \multirow[t]{2}{*}{11} & OD & $A R$ & $A R$ & $A R$ & $A R$ & $A R$ & $A R$ & $A R$ & $A R$ \\
\hline & OE & 15 & 15 & 5 & 0 & 5 & 10 & 20 & 20 \\
\hline \multirow[t]{2}{*}{12} & OD & 15 & 10 & 15 & 5 & 5 & 10 & 20 & 25 \\
\hline & $O E$ & $A R$ & $A R$ & $A R$ & $A R$ & $A R$ & $A R$ & $A R$ & $A R$ \\
\hline \multirow[t]{2}{*}{13} & OD & $A R$ & $A R$ & $A R$ & $A R$ & $A R$ & $A R$ & $A R$ & $A R$ \\
\hline & $O E$ & 20 & 15 & 5 & 5 & 10 & 15 & 20 & 15 \\
\hline \multirow[t]{2}{*}{14} & $O D$ & 5 & 5 & 5 & 10 & 5 & 5 & 15 & 5 \\
\hline & $O E$ & $A R$ & $A R$ & $A R$ & $A R$ & $A R$ & $A R$ & $A R$ & $A R$ \\
\hline \multirow[t]{2}{*}{15} & O D & 10 & 10 & 5 & 0 & 0 & 0 & 5 & 0 \\
\hline & $O E$ & $A R$ & $A R$ & $A R$ & $A R$ & $A R$ & $A R$ & $A R$ & $A R$ \\
\hline \multirow[t]{2}{*}{16} & $O D$ & 5 & 5 & 5 & 5 & 0 & 0 & 5 & 5 \\
\hline & $O E$ & $A R$ & $A R$ & $A R$ & $A R$ & $A R$ & $A R$ & $A R$ & $A R$ \\
\hline \multirow[t]{2}{*}{17} & OD & 95 & 100 & 120 & 120 & $A R$ & $A R$ & $A R$ & $A R$ \\
\hline & $O E$ & 15 & 15 & 10 & 0 & 0 & 0 & 10 & 5 \\
\hline \multirow[t]{2}{*}{18} & OD & 95 & 100 & 100 & 105 & 105 & 120 & $A R$ & $A R$ \\
\hline & $O E$ & 20 & 10 & 5 & 5 & 0 & 5 & 0 & 10 \\
\hline \multirow[t]{2}{*}{19} & OD & 100 & 100 & 105 & 110 & $A R$ & $A R$ & $A R$ & $A R$ \\
\hline & $O E$ & 15 & 10 & 5 & 5 & 10 & 5 & 5 & 0 \\
\hline \multirow[t]{2}{*}{20} & $O D$ & 100 & 110 & 110 & $A R$ & $A R$ & $A R$ & $A R$ & $A R$ \\
\hline & $O E$ & 10 & 5 & 5 & 10 & 5 & 0 & 5 & 10 \\
\hline \multirow[t]{2}{*}{21} & OD & $A R$ & $A R$ & $A R$ & $A R$ & AR & $A R$ & $A R$ & $A R$ \\
\hline & $O E$ & 5 & 10 & 10 & 5 & 15 & 15 & 20 & 5 \\
\hline \multirow[t]{2}{*}{22} & $O D$ & 15 & 10 & 10 & 5 & 5 & 10 & 20 & 15 \\
\hline & $O E$ & $A R$ & $A R$ & $A R$ & $A R$ & $A R$ & $A R$ & $A R$ & $A R$ \\
\hline
\end{tabular}

* AR: Ausência de resposta na intensidade máxima do equipamento.

Q uanto às medidas de imitância acústica, em todos os casos, foram obtidas curvas timpanométricas do tipo $\mathrm{A}$ em ambas as orelhas. No entanto, em $32 \%$ da população estudada, foi constatada a ausência do reflexo acústico modo contralateral da orelha normal em todas as freqüências pesquisadas (Casos 4, 7, 12, 16, 17, 20 e 21). Esta alteração do reflexo acústico foi irrelevante, pois este dado não interferiu no propósito do presente estudo.

$\mathrm{Na}$ ABR, avaliando-se as orelhas normais dos 22 casos deste estudo, obtivemos $100 \%$ de presença de limiares eletrofisiológicos dentro dos padrões da normalidade (menor ou igual a $25 \mathrm{~dB} N \mathrm{NA}$ - Tabela 2), sendo que em $95,5 \%$ destas orelhas as latências absolutas das Ondas I, III e V e latências interpicos I-III, III-V e I-V encontravam-se normais. Em um dos casos (Caso 21), 4,5\% restantes, observou-se atraso das latências absolutas das $O$ ndas III e V e conseqüente aumento das latências interpicos I-III e I-V sendo estes valores sugestivos de presença de alteração na via auditiva do tronco encefálico alto. Este aumento das latências absolutas das Ondas III e V não interferiu no intento deste estudo, pois 
Tabela 2. Valores dos limiares eletrofisiológicos (dB NA) de via aérea obtidos na audiometria de tronco encefálico, na ausência e presença do mascaramento contralateral, intensidades mínimas de mascaramento necessária empregado na orelha normal para testar a orelha comprometida à 90 dB NA e atenuação interaural para clicks obtidos dos 22 casos estudados.

\begin{tabular}{|c|c|c|c|c|c|}
\hline Casos & Orelha & $\begin{array}{c}\text { Limiar } \\
\text { Eletrofisiológico } \\
\text { Mascaramento } \\
\text { Ausente }\end{array}$ & $\begin{array}{c}\text { Limiar } \\
\text { Eletrofisiológico } \\
\text { Mascaramento } \\
\text { Presente }\end{array}$ & $\begin{array}{l}\text { Intensidade } \\
\text { Mínima de } \\
\text { Mascaramento } \\
\text { Necessário }\end{array}$ & $\begin{array}{c}\text { Atenuação } \\
\text { Interaural } \\
\text { para } \\
\text { Clicks }\end{array}$ \\
\hline \multirow[t]{2}{*}{1} & OD & 85 & $\mathrm{AR}^{*}$ & $35 \mathrm{~dB}$ & $65 \mathrm{~dB}$ \\
\hline & $\mathrm{OE}$ & 20 & ---- & & \\
\hline \multirow[t]{2}{*}{2} & OD & 70 & $\mathrm{AR}$ & $35 \mathrm{~dB}$ & $65 \mathrm{~dB}$ \\
\hline & $O E$ & 5 & ---- & & \\
\hline \multirow[t]{2}{*}{3} & OD & 80 & AR & $30 \mathrm{~dB}$ & $70 \mathrm{~dB}$ \\
\hline & $\mathrm{OE}$ & 10 & ---- & & \\
\hline \multirow[t]{2}{*}{4} & OD & 80 & AR & $35 \mathrm{~dB}$ & $65 \mathrm{~dB}$ \\
\hline & $O E$ & 15 & ---- & & \\
\hline \multirow[t]{2}{*}{5} & OD & 20 & ---- & $35 \mathrm{~dB}$ & $65 \mathrm{~dB}$ \\
\hline & $O E$ & 85 & AR & & \\
\hline \multirow[t]{2}{*}{6} & OD & 85 & AR & $25 \mathrm{~dB}$ & $75 \mathrm{~dB}$ \\
\hline & $\mathrm{OE}$ & 10 & ---- & & \\
\hline \multirow[t]{2}{*}{7} & OD & 20 & ---- & $35 \mathrm{~dB}$ & $65 \mathrm{~dB}$ \\
\hline & $O E$ & 85 & AR & & \\
\hline \multirow[t]{2}{*}{8} & OD & 85 & AR & $35 \mathrm{~dB}$ & $65 \mathrm{~dB}$ \\
\hline & $O E$ & 20 & ---- & & \\
\hline \multirow[t]{2}{*}{9} & OD & 90 & AR & $30 \mathrm{~dB}$ & $70 \mathrm{~dB}$ \\
\hline & $\mathrm{OE}$ & 20 & ---- & & \\
\hline \multirow[t]{2}{*}{10} & OD & 85 & AR & $35 \mathrm{~dB}$ & $65 \mathrm{~dB}$ \\
\hline & $\mathrm{OE}$ & 20 & ---- & & \\
\hline \multirow[t]{2}{*}{11} & OD & 90 & AR & $25 \mathrm{~dB}$ & $75 \mathrm{~dB}$ \\
\hline & $\mathrm{OE}$ & 15 & ---- & & \\
\hline \multirow[t]{2}{*}{12} & OD & 25 & ---- & $35 \mathrm{~dB}$ & $65 \mathrm{~dB}$ \\
\hline & $O E$ & 90 & AR & & \\
\hline \multirow[t]{2}{*}{13} & OD & 85 & $A R$ & $30 \mathrm{~dB}$ & $70 \mathrm{~dB}$ \\
\hline & $O E$ & 15 & ---- & & \\
\hline \multirow[t]{2}{*}{14} & OD & 25 & ---- & $35 \mathrm{~dB}$ & $65 \mathrm{~dB}$ \\
\hline & $\mathrm{OE}$ & 90 & AR & & \\
\hline \multirow[t]{2}{*}{15} & OD & 20 & ---- & $35 \mathrm{~dB}$ & $65 \mathrm{~dB}$ \\
\hline & $\mathrm{OE}$ & 85 & AR & & \\
\hline \multirow[t]{2}{*}{16} & OD & 15 & ---- & $35 \mathrm{~dB}$ & $65 \mathrm{~dB}$ \\
\hline & $\mathrm{OE}$ & 80 & AR & & \\
\hline \multirow[t]{2}{*}{17} & OD & 85 & AR & $35 \mathrm{~dB}$ & $65 \mathrm{~dB}$ \\
\hline & $\mathrm{OE}$ & 20 & ---- & & \\
\hline \multirow[t]{2}{*}{18} & OD & 85 & AR & $35 \mathrm{~dB}$ & $65 \mathrm{~dB}$ \\
\hline & $\mathrm{OE}$ & 20 & ---- & & \\
\hline \multirow[t]{2}{*}{19} & OD & 80 & AR & $30 \mathrm{~dB}$ & $70 \mathrm{~dB}$ \\
\hline & $\mathrm{OE}$ & 10 & ---- & & \\
\hline \multirow[t]{2}{*}{20} & OD & 85 & AR & $35 \mathrm{~dB}$ & $65 \mathrm{~dB}$ \\
\hline & $O E$ & 20 & ---- & & \\
\hline \multirow[t]{2}{*}{21} & OD & 85 & AR & $35 \mathrm{~dB}$ & $65 \mathrm{~dB}$ \\
\hline & $O E$ & 20 & ---- & & \\
\hline \multirow[t]{2}{*}{22} & OD & 15 & ---- & $25 \mathrm{~dB}$ & $75 \mathrm{~dB}$ \\
\hline & $\mathrm{OE}$ & 90 & AR & & \\
\hline
\end{tabular}

*AR: Ausência de resposta na intensidade máxima do equipamento.

nas avaliações subseqüentes os resultados comportaram-se de maneira semelhante aos outros casos avaliados.

Ao realizarmos a ABR nas orelhas comprometidas na ausência do mascaramento contralateral, observou-se a presença da Onda V com atraso da latência absoluta em todos os casos e nos seguintes limiares eletrofisiológicos: 70
dB NA (4,5\%), 80 dB NA (18\%), 85 dB NA (54,5\%) e $90 \mathrm{~dB}$ NA (23\%) (Tabela 2). Na intensidade de $90 \mathrm{~dB}$ NA (intensidade máxima do equipamento) todos os indivíduos apresentaram presença da O nda V com latência absoluta média de 7,27 milissegundos e amplitude média de 0,32 ( $\mu$ V) (Tabela 3). 
Tabela 3. Valores médios da latência absoluta e amplitude da onda $V$ obtidos com $90 \mathrm{~dB}$ NA de intensidade de estímulo acústico (click) na orelha comprometida, na ausência do mascaramento contralateral.

\begin{tabular}{ccc}
\hline Dados estatísticos & Latência Absoluta $(\mathrm{ms})$ & Amplitude $(\mu \mathrm{V})$ \\
\hline Média & 7,27 & 0,32 \\
Desvio Padrão & 0,29 & 0,12 \\
Tamanho & 22 & 22 \\
Limite Inferior & 7,15 & 0,26 \\
Limite Superior & 7,39 & 0,37 \\
\hline
\end{tabular}

Intervalo de Confiança (IC) $95 \%$

Tabela 4. Distribuição percentual dos valores de atenuação interaural para clicks ocorridos neste estudo.

\begin{tabular}{ccc}
\hline Atenuação Interaural & Número de casos & $\%$ \\
\hline $65 \mathrm{~dB}$ & 15 & $68,2 \%^{*}$ \\
$70 \mathrm{~dB}$ & 4 & $18,2 \%$ \\
$75 \mathrm{~dB}$ & 3 & $13,6 \%$ \\
Total & 22 & $100,0 \%$ \\
\hline
\end{tabular}

${ }^{*} \mathrm{P}<0,001$

A ocorrência da atenuação interaural para clicks de $65 \mathrm{~dB}$ foi superior as de $70 \mathrm{~dB}$ e $75 \mathrm{~dB}$ (Tabela 2), sendo que houve diferença estatisticamente significante entre a atenuação de $65 \mathrm{~dB}$ e as atenuações de $70 \mathrm{~dB}$ e $75 \mathrm{~dB}$ (Tabela 4). 0 valor da atenuação interaural mínima para clicks de $65 \mathrm{~dB}$ encontrado neste estudo mostrou-se semelhante ao obtido por Chiappa, Gladstone \& Young (1979) e Ozdamar \& Stein (1981). No entanto, em outros trabalhos, as atenuações interaurais encontradas foram de: $50 \mathrm{~dB}$ (Smyth, 1985) e 70-75 dB (Humes \& Ochs, 1982). Baseado nos diferentes valores de atenuação interaural para clicks encontrados neste estudo e na literatura pesquisada, evidenciamos a importância de que se estabeleça valores de atenuações interaurais para cada equipamento utilizado nos consultórios e clínicas.

Ao reavaliarmos as orelhas comprometidas através da $A B R$, aplicando-se o mascaramento contralateral, observamos que as $\mathrm{Ondas} V$ anteriormente visualizadas encontravam-se ausentes na intensidade máxima do estímulo acústico do equipamento (90 dB NA) (Tabela 2), sendo que a intensidade do mascaramento necessária foi de $10 \mathrm{~dB}$ acima da chegada da atenuação interaural em todos os casos. Desta maneira, pudemos concluir que os limiares eletrofisiológicos captados primeiramente, sem o uso do mascaramento, eram respostas advindas da orelha normal. A partir destes resultados pudemos comprovar que o uso concomitante do mascaramento na avaliação da audição por meio da ABR é um procedimento necessário ao examinar indivíduos com assimetria de limiares auditivos (iguais ou maiores do que $65 \mathrm{~dB}$ ) para a obtenção de resultados fidedignos. Estes achados mostram-se concordantes com a literatura pesquisada ${ }^{11-14}$. O utros autores, corroborando com nossos resultados, afirmaram que o mascaramento é necessário sempre que for utilizado estímulo acústico com intensidade superior a $70 \mathrm{~dB} \mathrm{NA}$, e quando a Onda I estiver ausente na presença da Onda $\mathrm{V}$ com atraso da latência absoluta ${ }^{1}$. Por outro lado, os resultados obtidos no presente estudo discordam com os achados dos trabalhos de Galambos \& Hecox (1978), Finito-Hieber, Hecox \& Cone (1979) e O wen \& Burkard (1991), os quais concluíram que o mascaramento contralateral não influenciava de maneira substancial na avaliação da ABR.

A Figura 1 representa as respostas eletrofisiológicas obtidas na ausência e na presença do mascaramento de um dos casos avaliados (Caso 10). Nas Figura 1-A e 1-B pode-se observar, respectivamente, o limiar eletrofisiológico da oreIha normal a $20 \mathrm{~dB} N A$ e da orelha comprometida a $85 \mathrm{~dB}$ NA na ausência do mascaramento. A atenuação interaural para este caso foi de $65 \mathrm{~dB}(85 \mathrm{~dB}-20 \mathrm{~dB})$. Vide que na audiometria tonal liminar há ausência de respostas da orelha comprometida na intensidade máxima do equipamento (105 dB NA para as freqüências de $250 \mathrm{~Hz}$ e $8000 \mathrm{~Hz}$ e $120 \mathrm{~dB}$ NA para as freqüências de $500 \mathrm{~Hz} \mathrm{a} 6000 \mathrm{~Hz}$ - Tabela 1). Ao retestarmos a orelha comprometida, por meio da $A B R$, no limiar eletrofisiológico antes encontrado (85 dB NA) simultaneamente a introdução do mascaramento contralateral à $30 \mathrm{~dB} W N(85 \mathrm{~dB}-65 \mathrm{~dB}+10 \mathrm{~dB})$ a resposta desaparece (Figura 1-C). Por fim, como mostrado na Figura 1-C, a intensidade do estímulo acústico (click) foi aumentada para 90 dB NA (intensidade máxima do aparelho) e concomitantemente foi aplicado o mascaramento contralateral na orelha normal à $35 \mathrm{~dB} W N(90 \mathrm{~dB}-65 \mathrm{~dB}+10 \mathrm{~dB})$. A resposta antes visualizada também foi ausentada. Esta ilustração mostra claramente a importância do uso do mascaramento para eliminar a resposta da via auditiva contralateral e obter assim um resultado fidedigno da avaliação da audição por meio da ABR em indivíduos portadores de perda auditiva neurossensorial unilateral de grau profundo.

As intensidades mínima e máxima necessárias de mascaramento para a avaliação da ABR neste estudo, levando-se em consideração o total de casos avaliados e a atenuação interaural para clicks individual, foi de respectivamente $25 \mathrm{~dB} W \mathrm{~N}$ (White Noise) e $35 \mathrm{~dB} W \mathrm{~W}$ (Tabela 2). Segundo a literatura pesquisada, a intensidade do mascaramento deve ser de 40 a $50 \mathrm{~dB} W \mathrm{~N}$ no ouvido não testado, para evitar a resposta eletrofisiológica contralateral ${ }^{1}$. Mediante estes resultados, sugerimos que a quantidade de mascaramento a ser utilizada seja determinada de acordo com o equipamento manuseado.

\section{CONCLUSÕES}

A partir da análise crítica dos resultados obtidos da avaliação da audição por meio da ABR em indivíduos portadores de perda auditiva neurossensorial unilateral de grau profundo, pudemos concluir que: 


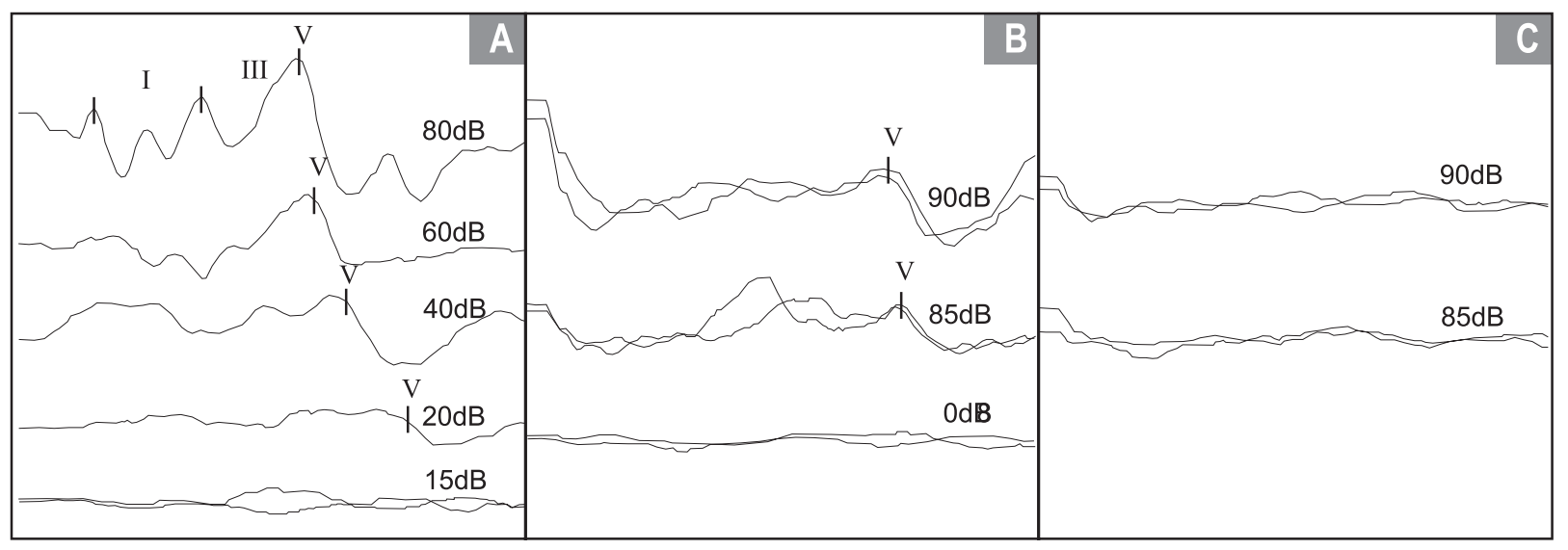

Figura 1. Audiometria de Tronco Encefálico na ausência e na presença do mascaramento contralateral (Caso 10). A:Limiar eletrofisiológico da orelha normal na ausência do mascaramento.

B:Limiar eletrofisiológico da orelha comprometida na ausência do mascaramento.

C:Ausência de respostas eletrofisiológicas da orelha comprometida na presença do mascaramento contralateral.

1. O mascaramento contralateral empregado na orelha normal é de extrema importância ao realizar a ABR em indivíduos portadores de perdas auditivas neurossensoriais unilaterais de grau profundo, para obtenção de resultados fidedignos.

2. A atenuação interaural para clicks foi maior $(65 \mathrm{~dB})$ do que a observada na audiometria tonal liminar, sendo necessário, portanto, uma menor intensidade de mascaramento para eliminar a resposta da via auditiva contralateral.

3. Os valores de atenuação interaural para clicks e a quantidade de mascaramento necessárias para a realização da ABR, em indivíduos portadores de perdas auditivas neurossensoriais unilaterais ou assimétricas, devem ser estabelecidos para cada clínica.

\section{REFERÊNCIAS BIBLIOGRÁFICAS}

1. Munhoz MSL, da Silva MLG, Caovilla HH, Frazza MM, Ganança MM, Câmera JLS. In: Munhoz MSL, Caovilla HH, da Silva MLG, Ganança MM. Audiologia Clínica; série Otoneurológica. Volume 2. São Paulo: Editora Atheneu; 2000. p.191- 230.

2. Lima MAMT. In: Frota S. Fundamentos em Fonoaudiologia: Audiologia. 1a edição Rio de Janeiro: Guanabara Kogaan; 1998. p. 147-60.

3. Musiek FE, Borenstein SP, Hall III JW \& Schwaber. In: Kat J. Tratado de Audiologia Clínica. 4a edição. São Paulo: Manole; 1999. p. 349-71.

4. Redondo MC. In: Frota S. Fundamentos em Fonoaudiologia: Audiologia. 1a edição. Rio de Janeiro: Guanabara Kogaan; 1998. p. 69-76.

5. Sanders JW \& Hall III JW. In: Musiek FE \& Rintelmann WF. Perspectivas Atuais em Avaliação Auditiva. 1a edição brasileira. Barueri: Manole; 2001. p. 63-84.
6. Goldstein BA \& Newman CW. In: Katz J. Tratado de Audiologia Clínica. 4a edição. São Paulo: Manole; 1999. p. 109-31.

7. Durrant JD \& Ferraro JA. In: Musiek FE \& Rintelmann WF. Perspectivas Atuais em Avaliação Auditiva. 1a edição brasileira. Barueri: Manole; 2001. p. 193-238.

8. Finitzo-Hieber T, Hecox K \& Cone B. Brain stem auditory evoked potentials in patients with congenital atresia. Laryngoscope 1979;89:1151-8.

9. Galambos R \& Hecox KE. Clinical applications of the auditory brainstem response. Otolaryngology Clinics of North American 1978;11:709-22.

10. Owen GA, \& Burkard R. Ipsilateral contralateral and binaural masking effects on the human brain-stem auditory-evoked responses to click stimuli. Journal of Acoustical Society of America 1991;89:1760-7.

11. Chiappa KH, Gladstone KJ \& Young RR. Studies of waveform variations in 50 normal human subjects. Arch Neurol 1979;36:817.

12. Ozdamar $O \&$ Stein L. Auditory brain stem response (ABR) in unilateral hearing loss. Laryngoscope 1981;41:565-74.

13. Humes LE \& Ochs MG. Use of contralateral masking in the measurement of the auditory brainstem response. Journal of Speech and Hearing Research 1982;25:528-35.

14. Smyth V. On the effect of cross-hearing and clinical masking on the auditory brain-stem evoked response. Electroencephalography and Clinical Neurophysiology 1985;61:26-9.

15. Mangabeira Albernaz $\mathrm{P}$, Mangabeira Albernaz PL, Mangabeira Albernaz LG e Mangabeira Albernaz FP. Otorrinolaringologia Prática. 10a edição. São Paulo: Savier; 1981.

16. Jerger J. Clinical experience with impedance audiometry. Arch Otolaryngol 1970;92:311-24.

17. Lopes Fo OC. Contribuição ao estudo da impedância acústica. São Paulo. Tese de Doutorado - Faculdade de Medicina da Universidade de São Paulo, 1972. 